\title{
Prioritized Sweeping: Reinforcement Learning With Less Data and Less Time
}

\author{
ANDREW W. MOORE \\ AWM@CS.CMU.EDU \\ CHRISTOPHER G. ATKESON \\ CGA@AI.MIT.EDU \\ MIT Artificial Intelligence Laboratory, NE43-771, 545 Technology Square, Cambridge, MA 02139
}

\begin{abstract}
We present a new algorithm, prioritized sweeping, for efficient prediction and control of stochastic Markov systems. Incremental learning methods such as temporal differencing and Q-learning have real-time performance. Classical methods are slower, but more accurate, because they make full use of the observations. Prioritized sweeping aims for the best of both worlds. It uses all previous experiences both to prioritize important dynamic programming sweeps and to guide the exploration of state-space. We compare prioritized sweeping with other reinforcement learning schemes for a number of different stochastic optimal control problems. It successfully solves large state-space real-time problems with which other methods have difficulty.
\end{abstract}

Keywords. Memory-based learning, learning control, reinforcement learning, temporal differencing, asynchronous dynamic programming, heuristic search, prioritized sweeping

\section{Introduction}

This article introduces a memory-based technique, prioritized sweeping, which can be used both for Markov prediction and reinforcement learning. Current, model-free, learning algorithms perform well relative to real time. Classical methods such as matrix inversion and dynamic programming perform well relative to the number of observations. Prioritized sweeping seeks to achieve the best of both worlds. Its closest relation from conventional $\mathrm{AI}$ is the search scheduling technique of the $A^{*}$ algorithm (Nilsson, 1971). It is a "memory-based" method (Stanfill \& Waltz, 1986) in that it derives much of its power from explicitly remembering all real-world experiences. Closely related research is being performed by Peng and Williams (1992) into a similar algorithm to prioritized sweeping, which they call Queue-Dyna.

We begin by providing a review of the problems and techniques in Markov prediction and control. More thorough reviews may be found in Sutton (1988), Barto et al. (1989), Sutton (1990), Kaelbling (1990), and Barto et al. (1991).

A discrete, finite Markov system has $S$ states. Time passes as a series of discrete clock ticks, and on each tick the state may change. The probability of possible successor states is a function only of the current system state. The entire system can thus be specified by $S$ and a table of transition probabilities.

$$
\begin{array}{cccc}
q_{11} & q_{12} & \cdots & q_{1 S} \\
q_{21} & q_{22} & \cdots & q_{2 S} \\
\vdots & \vdots & & \vdots \\
q_{S 1} & q_{S 2} & \cdots & q_{S S}
\end{array}
$$




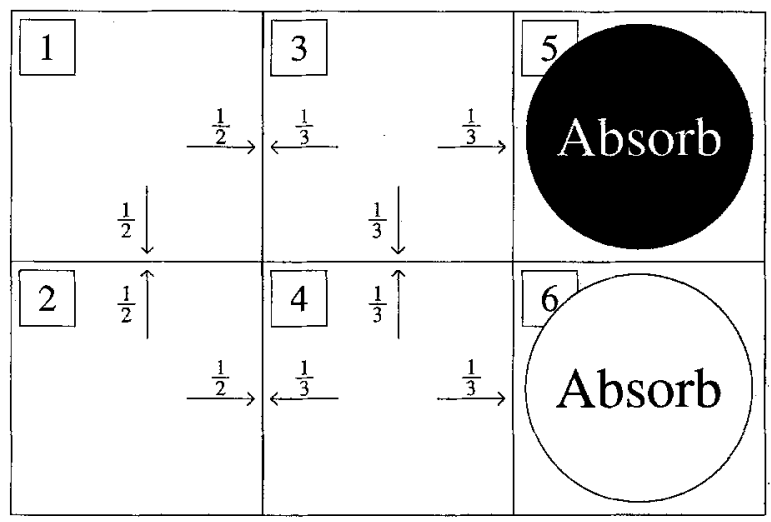

Figure 1. A six-state Markov system.

where $q_{i j}$ denotes the probability that, given we are in state $i$, we will be in state $j$ on the next time step. The table must satisfy $\Sigma_{j=1}^{S} q_{i j}=1$ for every $i$.

Figure 1 shows an example with six states corresponding to the six cells. With the exception of the rightmost states, on each time step the system moves at random to a neighbor. For example, state 1 moves directly to state 3 with probability $1 / 2$, and thus $q_{13}=1 / 2$.

The state-space of a Markov system is partitioned into two subsets: the non-terminal states, NONTERMS, and the terminal states, TERMS. Once a terminal state is entered, it is never left $\left(k \in\right.$ TERMS $\Rightarrow q_{k k}=1$ ). In the example, the two rightmost states are terminal.

A Markov system is defined as absorbing if from every non-terminal state it is possible to eventually enter a terminal state. We restrict our attention to absorbing Markov systems.

Let us first consider questions such as, "Starting in state $i$, what is the probability of eventual absorption by terminal state $k$ ?" Write this value as $\pi_{i k}$. All the absorption probabilities for terminal state $k$ can be computed by solving the following set of linear equations. Assume that the non-terminal states are indexed by $1,2, \ldots, S_{n t}$ where $S_{n t}$ is the number of non-terminals.

$$
\begin{array}{cc}
\pi_{1 k}=q_{1 k}+q_{11} \pi_{1 k}+q_{12} \pi_{2 k}+\cdots+q_{1 S_{n t}} \pi_{S_{n t} k} \\
\pi_{2 k}=q_{2 k}+q_{21} \pi_{2 k}+q_{22} \pi_{2 k}+\cdots+q_{2 S_{n t}} \pi_{S_{n t} k} \\
\vdots \quad \vdots & \\
\pi_{S_{n t} k} & =q_{S_{n k} k}+q_{S_{n t} \pi_{1 k}}+q_{S_{n t} 2} \pi_{2 k}+\cdots+q_{S_{n t} S_{n t} \pi_{S_{n t} k}}
\end{array}
$$

When the transition probabilities $\left\{q_{i j}\right\}$ are known, it is thus an easy matter to compute the eventual absorption probabilities. Machine learning can be applied to the case in which the transition probabilities are not known in advance, and all we may do instead is watch a series of state transitions. Such a series is normally arranged into a set of trials-each trial starts in some state and then continues until the system enters a terminal state. In our example, the learner might be shown 


$$
\begin{gathered}
3 \rightarrow 4 \rightarrow 3 \rightarrow 1 \rightarrow 2 \rightarrow 4 \rightarrow 6 \\
3 \rightarrow 5 \\
\quad 1 \rightarrow 2 \rightarrow 1 \rightarrow 3 \rightarrow 5
\end{gathered}
$$

Learning approaches to this problem have been widely studied. A recent contribution of great relevance is an elegant algorithm called Temporal Differencing (Sutton, 1988).

\subsection{The temporal differencing algorithm reviewed}

We describe the discrete state-space case of the temporal differencing (TD) algorithm. TD can, however, also be applied to systems with continuous state-spaces in which long-term probabilities are represented by parametric function approximators such as neural networks (Tesauro, 1991).

The prediction process runs in a series of epochs. Each epoch ends when a terminal state is entered. Assume we have passed through states $i_{1}, i_{2}, \ldots i_{n}, i_{n+1}$ so far in the current epoch. $n$ is our age within the epoch and $t$ is our global age. $i_{n} \rightarrow i_{n+1}$ is the most recently observed transition. Let $\hat{\pi}_{i k}[t]$ be the estimated value of $\pi_{i k}$ after the system has been running for $t$ state-transition observations. Then the TD algorithm for discrete statespaces updates these estimates according to the following rule:

for each $i \in$ NONTERMS (the set of non-terminal states)

$$
\begin{aligned}
& \text { for each } k \in \text { TERMS (the set of terminal states) } \\
& \hat{\pi}_{i k}[t+1]=\hat{\pi}_{i k}[t]+\alpha\left(\hat{\pi}_{i_{n+1} k}[t]-\hat{\pi}_{i_{n} k}[t]\right) \sum_{j=1}^{n} \lambda^{n-j} X_{i}\left(i_{j}\right)
\end{aligned}
$$

where $\alpha$ is a learning state parameter $0<\alpha<1, \lambda$ is a memory constant $0 \leq \lambda \leq 1$, and

$$
X_{i}\left(i_{j}\right)= \begin{cases}1 & \text { if } i_{j}=i \\ 0 & \text { otherwise }\end{cases}
$$

In practice there is a computational trick that requires considerably less computation than the algorithm of equation (4) but which computes the same values (Sutton, 1988). The TD algorithm then requires $O\left(S_{t}\right)$ computation steps per real observation, where $S_{t}$ is the number of terminal states. Convergence proofs exist for several formulations of the TD algorithm (Sutton, 1988; Dayan, 1992). 


\subsection{The classical approach}

The classical method proceeds by building a maximum likelihood model of the state transitions. $q_{i j}$ is estimated by

$$
\hat{q}_{i j}=\frac{\text { Number of observations } i \rightarrow j}{\text { Number of occasions in state } i}
$$

After $t+1$ observations, the new absorption probability estimates are computed to satisfy, for each terminal state $k$, the $S_{n t} \times S_{n t}$ linear system

$$
\hat{\pi}_{i k}[t+1]=\hat{q}_{i k}+\sum_{j \in \operatorname{succs}(i) \text { กNONTERMS }} \hat{q}_{i j} \hat{\pi}_{j k}[t+1]
$$

where $\operatorname{succs}(i)$ is the set of all states which have been observed as immediate successors of $i$ and NONTERMS is the set of non-terminal states. It is clear that if the $\hat{q}_{i k}$ estimates were correct, then the solution of equation (7) would be the solution of equation (2).

Notice that the values $\hat{\pi}_{i k}[t+1]$ depend only on the values of $\hat{q}_{i k}$ after $t+1$ observations - they are not defined in terms of the previous absorption probability estimates $\hat{\pi}_{i k}[t]$. However, it is efficient to solve equation (7) iteratively. Let $\left\{\rho_{i k}\right\}$ be a set of intermediate iteration variables containing intermediate estimates of $\hat{\pi}_{i k}[t+1]$. What initial estimates should be used to start the iteration? An excellent answer is to use the previous absorption probability estimates $\hat{\pi}_{i k}[t]$.

The complete algorithm, performed once after every real-world observation, is shown in figure 2. The transformation on the $\rho_{i k}$ 's can be shown to be a contraction mapping as defined in section 3.1 of Bertsekas and Tsitsiklis (1989), and thus, as the same reference proves, convergence to a solution satisfying equation (7) is guaranteed. If, according to the estimated transitions, all states can reach a terminal state, then this solution is unique. The inner loop ("for each $k \in$ TERMS ...") is referred to as a probability backup operation, and requires $O\left(S_{t} \mu_{\text {succs }}\right)$ basic operations, where $\mu_{\text {succs }}$ is the mean number of observed stochastic successors.

1. for each $i \in$ NONTERMS, for each $k \in$ TERMS,

2. repeat

$$
\rho_{i k}:=\hat{\pi}_{i k}[t]
$$

$2.1 \Delta_{\max }:=0$

2.2 for each $i \in$ NONTERMS

for each $k \in$ TERMS

$$
\begin{gathered}
\rho_{\text {new }}=\hat{q}_{i k}+\Sigma_{j \in \operatorname{Succs}(i)} \hat{q}_{i j} \rho_{j k} \\
\Delta:=\left|\rho_{\text {new }}-\rho_{i k}\right| \\
\rho_{i k}:=\rho_{\text {new }} \\
\Delta_{\max }:=\max \left(\Delta_{\max }, \Delta\right)
\end{gathered}
$$

until $\Delta_{\max }<\epsilon$

3. for each $i \in$ NONTERMS, for each $k \in$ TERMS

$$
\tilde{\pi}_{i k}[t+1]:=\rho_{i k}
$$

Figure 2. Stochastic prediction with full Gauss-Seidel iteration. 
Gauss-Seidel is an expensive algorithm, requiring $O\left(S_{n t}\right)$ backups per real-world observation for the inner loop 2.2 alone. The absorption predictions before the most recent observation, $\hat{\pi}_{i k}[t]$, normally provide an excellent initial approximation, and only a few iterations are required. However, when an "interesting" observation is encountered-for example, a previously never-experienced transition to a terminal state-many iterations, perhaps more than $S_{n t}$, are needed for convergence.

\section{Prioritized sweeping}

Prioritized sweeping is designed to perform the same task as Gauss-Seidel iteration while using careful bookkeeping to concentrate all computational effort on the most "interesting" parts of the system. It operates in a similar computation regime as the Dyna architecture (Sutton, 1990), in which a fixed, but non-trivial, amount of computation is allowed between each real-world observation. Peng and Williams (1992) are exploring a closely related approach to prioritized sweeping, developed from Dyna and Q-learning (Watkins, 1989).

Prioritized sweeping uses the $\Delta$ value from the probability update step 2.2 in the previous algorithm to determine which other updates are likely to be "interesting"-if the step produces a large change in the state's absorption probabilities, then it is interesting because it is likely that the absorption probabilities of the predecessors of the state will change. If, on the other hand, the step produces a small change, then we will assume that there is less urgency to process the predecessors. The predecessors of a state $i$ are all those states $i^{\prime}$ that have, at least once in the history of the system, performed a one-step transition $i^{\prime} \rightarrow i$.

If we have just changed the absorption probabilities of $i$ by $\Delta$ then the maximum possible one-processing-step change in predecessor $i^{\prime}$ caused by our change in $i$ is $\hat{q}_{i^{\prime}} \Delta$. This value is the priority $P$ of the predecessor $i^{\prime}$, and if $i^{\prime}$ is not currently on the priority queue it is placed there at priority $P$. If it $i s$ already on the queue, but at lower priority, then it is promoted.

After each real-world observation $i \rightarrow j$, the transition probability estimate $\hat{q}_{i j}$ is updated along with the probabilities of transition to all other previously observed successors of $i$. Then state $i$ is promoted to the top of the priority queue so that its absorption probabilities are updated immediately. Next, we continue to process further states from the top of the queue. Each state that is processed may result in the addition or promotion of its predecessors within the queue. This loop continues for a preset number of processing steps or until the queue empties.

Thus if a real-world observation is interesting, all its predecessors and their earlier ancestors quickly find themselves near the top of the priority queue. On the other hand, if the real-world observation is unsurprising, then the processing immediately proceeds to other, more important areas of state-space that had been under consideration on previous time steps. These other areas may be different from those in which the system currently finds itself.

Let us look at the formal algorithm in figure 3. On entry we assume the most recent state transition was from $i_{\text {recent }}$. We drop the $[t]$ suffix from the $\hat{\pi}_{i k}[t]$ notation. 
1. Promote state $i_{\text {recent }}$ to top of priority queue.

2. While we are allowed further processing and priority queue not empty

2.1 Remove the top state from the priority queue. Call it $i$

$2.2 \Delta_{\max }=0$

2.3 for each $k \in$ TERMS

$$
\begin{gathered}
\rho_{\text {new }}=\hat{q}_{i k}+\sum \hat{q}_{i j} \pi_{j k} \\
\Delta:=\left|\rho_{\text {new }}-\hat{\pi}_{i k}\right| \\
\hat{\pi}_{i k}:=\rho_{\text {new }} \\
\Delta_{\text {max }}:=\max \left(\Delta_{\max }, \Delta\right)
\end{gathered}
$$

2.4 for each $i^{\prime} \in \operatorname{preds}(i)$

$P:=\hat{q}_{i^{\prime} i} \Delta_{\max }$

If $P>\epsilon$ (a tiny threshold) and if ( $i$ ' is not on queue or $P$ exceeds the current priority of $i^{\prime}$ ) then promote $i^{\prime}$ to new priority $P$.

Figure 3. The prioritized sweeping algorithm.

The decision of when we are allowed further processing, at the start of step 2, could be implemented in many ways. In our subsequent experiments, the rule is simply that a maximum of $\beta$ backups are permitted per real-world observation.

There are many possible priority queue implementations, including a heap (Knuth, 1973), which was used in all experiments in this article. The cost of the algorithm is

$$
O\left(\beta S_{t}\left(\mu_{\text {succs }}+\mu_{\text {preds }} \operatorname{PQCOST}\left(S_{n t}\right)\right)\right)
$$

basic operations, where at most $\beta$ states are processed from the priority queue and $\mathrm{PQCOST}(N)$ is the cost of accessing a priority queue of length $N$. For the heap implementation, this is $\log _{2} N$.

States are only added to the queue if their priorities are above a tiny threshold $\epsilon$. This is a value close to the machine floating-point precision. Stopping criteria are fraught with danger, but in this article we discuss such dangers no further except to note that in our experiments they have caused no problems.

Prioritized sweeping is a heuristic, and in this article no formal proof of convergence, or convergence rate, is given. We expect to be able to prove convergence using techniques from asynchronous Dynamic Programming (Bertsekas \& Tsitsiklis, 1989) and variants of the temporal differencing analysis of Dayan (1992). Later, this article gives some empirical experiments in which convergence is relatively quick.

The memory requirements of learning the $S \times S$ transition probability matrix, where $S$ is the number of states, may initially appear prohibitive, especially since we intend to operate with more than 10,000 states. However, we need only allocate memory for the experiences the system actually has, and for a wide class of physical systems there is not enough time in the lifetime of the system to run out of memory.

Similarly, the average number of successors and predecessors of states in the estimated transition matrix can be assumed $\ll S$. A simple justification is that few real problems are fully connected, but a deeper reason is that for large $S$, even if the true transition probability matrix is not sparse, there will never be time to gain enough experience for the estimated transition matrix to not be sparse. 


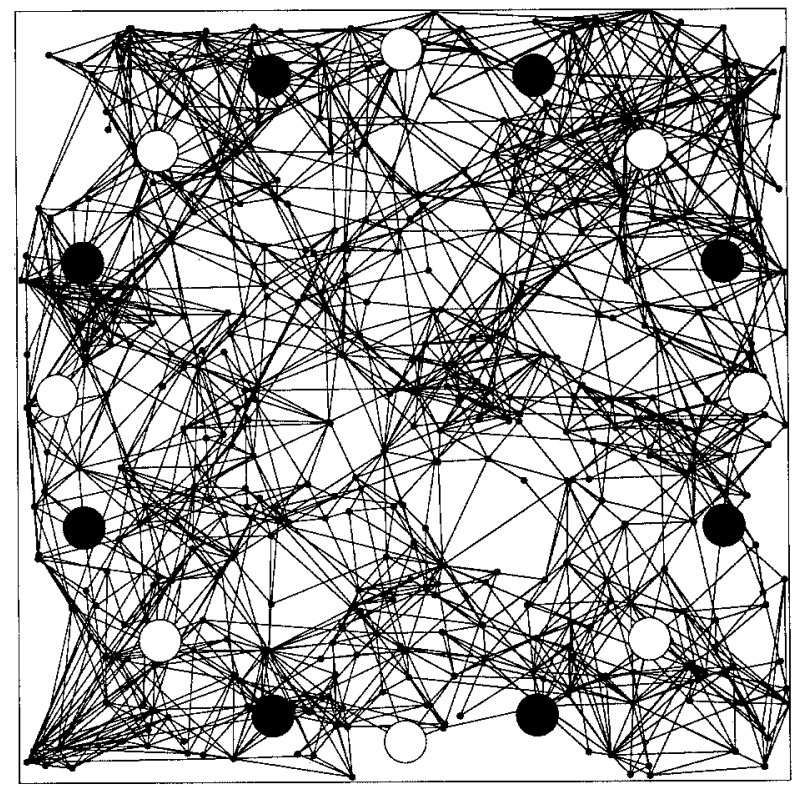

Figure 4. A 500-state Markov system. Each state has, on average, five stochastic successors.

\section{A Markov prediction experiment}

Consider the 500 state Markov system depicted in figure 4, which is a more complex version of the problem presented in figure 1. The appendix gives details of how this problem was randomly generated. The system has 16 terminal states, depicted by white and black circles. The prediction problem is to estimate, for every non-terminal state, the long-term probability that it will terminate in a black, rather than a white, circle. The data available to the learner comprise a sequence of observed state transitions.

Temporal differencing, the classical method, and prioritized sweeping were all applied to this problem. Each learner was shown the same sequence of state transitions. TD used parameters $\lambda=0.25$ and $\alpha=0.05$, which gave the best performance of a number of manually optimized experiments. The classical method was required to compute up-todate absorption probability estimates after every real-world observation. Prioritized sweeping was allowed five backups per real experience $(\beta=5)$; it thus updated the $\hat{\pi}_{i k}$ estimates for the five highest priority states between each real-world observation. The threshold for ignoring tiny changes, $\epsilon$, was $10^{-5}$. Each method was evaluated at a number of stages of learning, by stopping the real time clock and computing the error between the estimated white-absorption probabilities, which we denote by $\hat{\pi}_{i, \text { WHITE }}$, and the true values, which

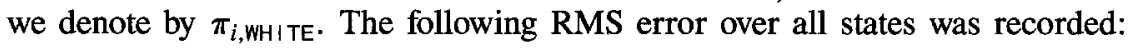

$$
\sqrt{\frac{1}{S_{n t}} \Sigma_{i=0}^{S_{n t}}\left(\pi_{i \mathrm{WHITE}}-\hat{\pi}_{i \mathrm{WHITE}}\right)^{2}}
$$




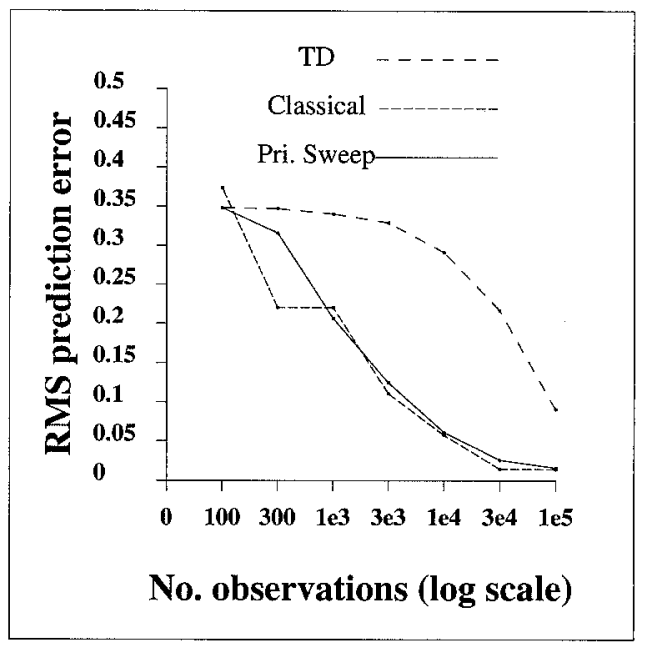

Figure 5. RMS prediction error between true absorption probabilities and predicted values, plotted against number of data-points observed. For prioritized sweeping, $\beta=5$, and $\epsilon=10^{-5}$.

In figure 5 we look at the RMS error plotted against the number of observations. After 100,000 experiences, all methods are performing well; TD is the weakest, but even it manages an RMS error of only 0.1 .

In figure 6 we look at a different measure of performance: prediction error plotted against computation time. Here we see the great weakness of the classical technique. Performing

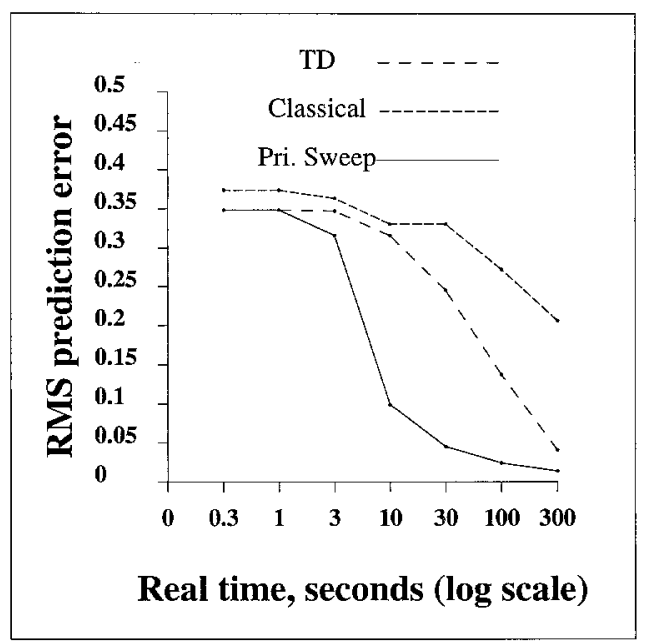

Figure 6. RMS prediction error between true absorption probabilities and predicted values, plotted against real time, in seconds, running the problem on a Sun- 4 workstation. 
Table 1. RMS prediction error: mean and standard deviation for ten experiments.

\begin{tabular}{lccr}
\hline & TD & Classical & Pri. Sweep \\
\hline After 100,000 observations & $0.14 \pm 0.077$ & $0.024 \pm 0.0063$ & $0.024 \pm 0.0061$ \\
After 300 seconds & $0.079 \pm 0.067$ & $0.23 \pm 0.038$ & $0.021 \pm 0.0080$ \\
\hline
\end{tabular}

the Gauss-Seidel algorithm of figure 2 after each observation gives excellent predictions but is very time consuming, and after 300 seconds there has only been time to process a few thousand observations. After the same amount of time, TD has had time to process almost half a million observations. Prioritized sweeping performs best relative to real time. It takes approximately ten times as long as TD to process each observation, but because the data are used more effectively, convergence is superior.

Ten further experiments, each with a different random 500 state problem, were run. These further runs, the final results of which are given in table 1 , indicate that the graphs of figures 5 and 6 are not atypical.

This example has shown the general theme of this article. Model-free methods perform well in real time but make weak use of their data. Classical methods make good use of their data but are often impractically slow. Techniques such as prioritized sweeping are interesting because they may be able to achieve the advantages of both.

There is an important footnote concerning the classical method. If the problem had only required that a prediction be made after all transitions had been observed, then the only real-time cost would have been recording the transitions in memory. The absorption probabilities could then have been computed as an individual large computation at the end of the sequence, giving the best possible estimate with a relatively small overall time cost. For the 500-state problem, we estimate the cost as approximately 30 seconds for 100,000 points. Prioritized sweeping could also benefit from only being required to predict after seeing all the data, although with little advantage over the simpler, classical algorithm. Prioritized sweeping is thus most usefully applicable to the class of tasks in which a prediction is required on every time step. Furthermore, the remainder of this article concerns control of Markov decision tasks, in which the maintenance of up-to-date predictions is particularly beneficial.

\section{Learning control of Markov decision tasks}

Let us consider a related stochastic prediction problem, which bridges the gap between Markov prediction and control. Suppose the system gets rewarded for entering certain states and gets punished for entering others. Let the reward of the $i$ th state be $r_{i}$. An important quantity is then the expected discounted reward-to-go of each state. This is an infinite sum of expected future rewards, with each term supplemented by an exponentially decreasing weighting factor $\gamma^{k}$ where $\gamma$ is called the discount factor. The expected discounted rewardto-go is 


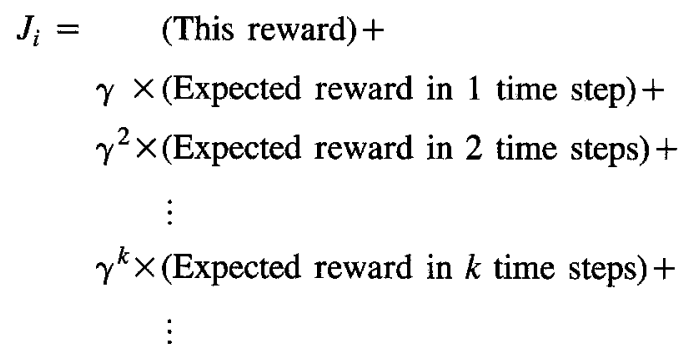

For each $i, J_{i}$ can be computed recursively as a function of its immediate successors.

$$
\begin{gathered}
J_{1}=r_{1}+\gamma \times\left(q_{11} J_{1}+q_{12} J_{2}+\cdots+q_{1 S} J_{S}\right) \\
J_{2}=r_{2}+\gamma \times\left(q_{21} J_{1}+q_{22} J_{2}+\cdots+q_{2 S} J_{S}\right) \\
\vdots \\
\vdots \\
J_{S}= \\
r_{S}+\gamma \times\left(q_{S 1} J_{1}+q_{S 2} J_{2}+\cdots+q_{S S} J_{S}\right)
\end{gathered}
$$

which is another set of linear equations that may be solved if the transition probabilities $q_{i j}$ are known. If they are not known, but instead a sequence of state transitions and $r_{i}$ observations is given, then slight modifications of TD, the classical algorithm, and prioritized sweeping can all be used to estimate $J_{i}$.

\section{Markov decision tasks}

Markov decision tasks are an extension of the Markov model in which, instead of passively watching the state move around randomly, we are able to influence it.

Associated with each state, $i$, is a finite, discrete set of actions, act i ons $(i)$. On each time step, the controller must choose an action. The probabilities of potential next states depend not only on the current state, but also on the chosen action. We will supplement our example problem with actions:

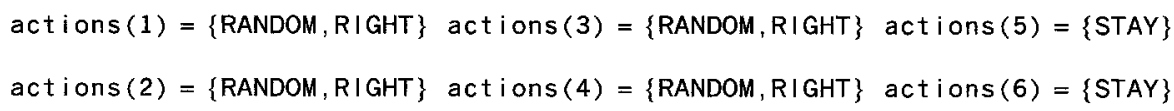

where RANDOM causes the same random transitions as before, RIGHT moves, with probability 1 , to the cell immediately to the right, and STAY makes us remain in the same state. There is still no escape from states 5 and 6.

We use the notation $q_{i j}^{a}$ for the probability that we move to state $j$, given that we have commenced in state $i$ and applied action $a$. Thus, in our example $q_{13}^{\text {RANDOM }}=1 / 2$ and $q_{13}^{\mathrm{R} \mid \mathrm{GHT}}=1$.

A policy is a mapping from states to actions. For example, figure 7 shows the policy

$$
\begin{aligned}
& 1 \rightarrow \text { RIGHT } 3 \rightarrow \text { RIGHT } 5 \rightarrow \text { STAY } \\
& 2 \rightarrow \text { RANDOM } 4 \rightarrow \text { RANDOM } 6 \rightarrow \text { STAY }
\end{aligned}
$$




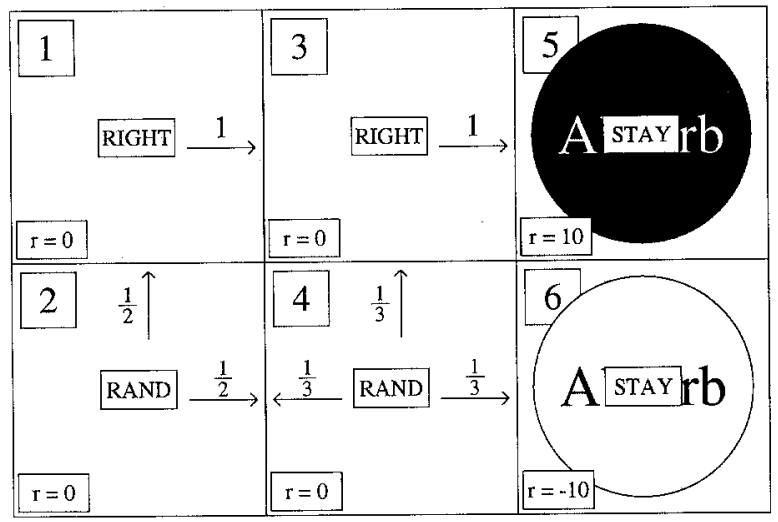

Figure 7. The policy defined by equation (13). Also shown is a reward function (bottom left of each cell). Large expected reward-to-go involves getting to ' 5 ' and avoiding ' 6 '.

If the controller chooses actions according to a fixed policy, then it behaves like a Markov system. The expected discounted reward-to-go can then be defined and computed in the same manner as equation (11).

If the goal is large reward-to-go, then some policies are better than others. An important result from the theory of Markov decision tasks tells us that there always exists at least one policy that is optimal in the following sense. For every state, the expected discounted reward-to-go using an optimal policy is no worse than that from any other policy.

Furthermore, there is a simple algorithm for computing both an optimal policy and the expected discounted reward-to-go of this policy. The algorithm is called Dynamic Programming (Bellman, 1957). It is based on the following relationship, known as Bellman's equation, which holds between the optimal expected discounted reward-to-go at different states.

$$
J_{i}=\max _{a \in \operatorname{actions}(i)}\left(r_{i}+\gamma\left(q_{i 1}^{a} J_{1}+q_{i 2}^{a} J_{2}+\cdots+q_{i S}^{a} J_{S}\right)\right)
$$

Dynamic programming applied to our example gives the policy shown in figure 7, which happens to be the unique optimal policy.

A very important question for machine learning has been how to obtain an optimal, or near optimal, policy when the $q_{i j}^{a}$ values are not known in advance. Instead, a series of actions, state transactions, and rewards is observed. For example:

$$
\begin{aligned}
& 1\left(r_{1}=0\right) \stackrel{\text { RANDOM }}{\rightarrow} 2\left(r_{2}=0 \stackrel{\text { RANDOM }}{\rightarrow} 4\left(r_{4}=0\right) \stackrel{\text { RIGHT }}{\rightarrow} 6\left(r_{6}=10\right)\right. \\
& 2\left(r_{2}=0\right) \stackrel{\text { RANDOM }}{\rightarrow} 1\left(r_{1}=0 \stackrel{\text { RIGHT }}{\rightarrow} 3\left(r_{3}=0\right) \stackrel{\text { RANDOM }}{\rightarrow} 5\left(r_{5}=-10\right)\right. \\
& 3\left(r_{3}=0\right) \stackrel{\text { RANDOM }}{\rightarrow} 5\left(r_{2}=10\right.
\end{aligned}
$$


A critical difference between this problem and the Markov prediction problem of the earlier sections is that the controller now affects which transitions are seen, because it supplies the actions.

The question of learning in such systems is studied by the field of reinforcement learning, which is also known as "learning control of Markov decision tasks." Early contributions to this field were the checkers player of Samuel (1959) and the BOXES system of Michie and Chambers (1968). Even systems that may at first appear trivially small, such as the two-armed bandit problem (Berry \& Fristedt, 1985) have promoted rich and interesting work in the statistics community.

The technique of gradient descent optimization of neural networks in combination with approximations to the policy and reward-to-go (called the "adaptive heuristic critic") was introduced by Sutton (1984). Kaelbling (1990) introduced several applicable techniques, including the Interval Estimation algorithm. Watkins (1989) introduced an important modelfree asynchronous Dynamic Programming technique called Q-learning. Sutton (1990) has extended this further with the Dyna architecture. Christiansen et al. (1990) applied a planner, closely related to Dynamic Programming, to a tray tilting robot. An excellent review of the entire field may be found in Barto et al. (1991).

\subsection{Prioritized sweeping for learning control of Markov decision tasks}

The main differences between this case and the previous application of prioritized sweeping are

1. We need to estimate the optimal discounted reward-to-go, $J$, of each state, rather than the eventual absorption probabilities.

2. Instead of using the absorption probability backup equation (6), we use Bellman's equation (Bellman, 1957; Bertsekas \& Tsitsiklis, 1989):

$$
\hat{J}_{i}=\max _{a \in \operatorname{actions}(i)}\left(\hat{r}_{i}^{a}+\gamma \times \sum_{j \in \operatorname{succs}(i, a)} \hat{q}_{i j}^{a} \hat{J}_{j}\right)
$$

where $\hat{J_{i}}$ is the estimate of the optimal discounted reward starting from state $i, \gamma$ is the discount factor, act i ons $(i)$ is the set of possible actions in state $i$, and $\hat{q}_{i j}^{a}$ is the maximum likelihood estimated probability of moving from state $i$ to state $j$ given that we have applied action $a$. The estimated immediate reward, $\hat{r}_{i}^{a}$, is computed as the mean reward experienced to date during all previous applications of action $a$ in state $i$.

3. The rate of learning can be affected considerably by the controller's exploration strategy.

The algorithm for prioritized sweeping in conjunction with Bellman's equation is given in figure 8 . The only substantial difference between this algorithm and the prediction case is the state backup step, namely, the application of Bellman's equation in step 2.2. Notice also that the predecessors of a state are now a set of state-action pairs. 
1. Promote state $i_{\text {recent }}$ to top of priority queue.

2. While we are allowed further processing and priority queue not empty

2.1 Remove the top state from the priority queue. Call it $i$

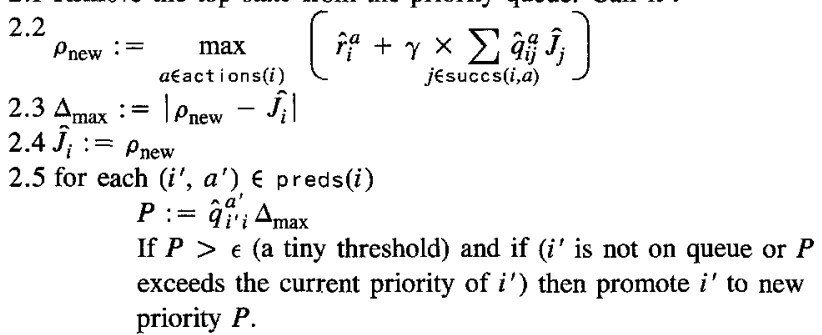

Figure 8. The prioritized sweeping algorithm for Markov decision tasks.

Let us now consider the question of how best to gain useful experience in a Markov decision task. The formally correct method would be to compute the exploration that maximizes the expected reward received over the robot's remaining life. This computation, which requires a prior probability distribution over the space of Markov decision tasks, is unrealistically expensive. It is computationally exponential in all of 1) the number of time steps for which the system is to remain alive, 2) the number of states in the system, and 3) the number of actions available (Berry \& Fristedt, 1985).

An exploration heuristic is thus required. Kaelbling (1990) and Barto et al. (1991) both give excellent overviews of the wide range of heuristics that have been proposed.

We use the philosophy of optimism in the face of uncertainty, a method successfully developed by the Interval Estimation (IE) algorithm of Kaelbling (1990) and by the exploration bonus technique in Dyna (Sutton 1990). The same philosophy is also used by Thrun and Möller (1992).

A slightly different heuristic is used with the prioritized sweeping algorithm. This is because of minor problems of computational expense for IE and the instability of the exploration bonus in large state-spaces.

The slightly different optimistic heuristic is as follows. In the absence of contrary evidence, any action in any state is assumed to lead us directly to a fictional absorbing state of permanently large reward $r^{\text {opt }}$. The amount of evidence to the contrary that is needed to quench our optimism is a system parameter, $T_{\text {bored }}$. If the number of occurrences of a given state-action pair is less than $T_{\text {bored, }}$, we assume that we will jump to a fictional state with subsequent long-term reward $r^{\mathrm{opt}}+\gamma r^{\mathrm{opt}}+\gamma^{2} r^{\mathrm{opt}}+\ldots=r^{\mathrm{opt}} /(1-\gamma)$. If the number of occurrences is more than $T_{\text {bored }}$, then we use the true, non-optimistic, assumption. Thus the optimistic reward-to-go estimate $\hat{J}^{\text {opt }}$ is

$$
\hat{J}_{i}^{\mathrm{opt}}=\max _{a \in \operatorname{actions}(i)} \begin{cases}r^{\mathrm{opt}} /(1-\gamma) & \text { if } n_{i}^{a}<T_{\text {bored }} \\ \hat{r}_{i}^{a}+\gamma \times \sum_{j \in \operatorname{succs}(i, a)} \hat{q}_{i j}^{a} \hat{J}_{j}^{\mathrm{opt}} & \text { otherwise }\end{cases}
$$


where $n_{i}^{a}$ is the number of times action $a$ has been tried to date in state $i$. The important feature, identified by Sutton (1990), is the planning to explore behavior caused by the appearance of the optimism on both sides of the equation. A related exploration technique was used by Christiansen et al. (1990). Consider the situation in figure 9. The top left-hand corner of state-space only looks attractive if we use an optimistic heuristic. The areas near the frontiers of little experience will have high $\hat{J}^{\text {opt }}$, and in turn the areas near those have nearly as high $\hat{J}^{\text {opt }}$. Therefore, if prioritized sweeping (or any other asynchronous dynamic programming method) does its job, from START we will be encouraged to go north towards the unknown instead of east to the best reward discovered to date.

The system parameter $r^{\text {opt }}$ does not require fine tuning. It can be set to a gross overestimate of the largest possible reward, and the system will simply continue exploration until it has sampled all state-action combinations $T_{\text {bored }}$ times. However, section 6 discusses its use as a search-guiding heuristic similar to the heuristic at the heart of $A^{*}$ search.

The $T_{\text {bored }}$ parameter, which defines how often we must try a given state-action combination before we cease our optimism, certainly does require forethought by the human programmer. If too small, we might overlook some low-probability but highly rewarding stochastic successor. If too high, the system will waste time needlessly resampling already reliable statistics. Thus, the exploration procedure does not have full autonomy. This is, arguably, a necessary weakness of any non-random exploration heuristic. Dyna's exploration bonus contains a similar parameter in the relative size of the exploration bonus compared to the expected reward, and Interval Estimation has the parameter implicit in the optimistic confidence level.

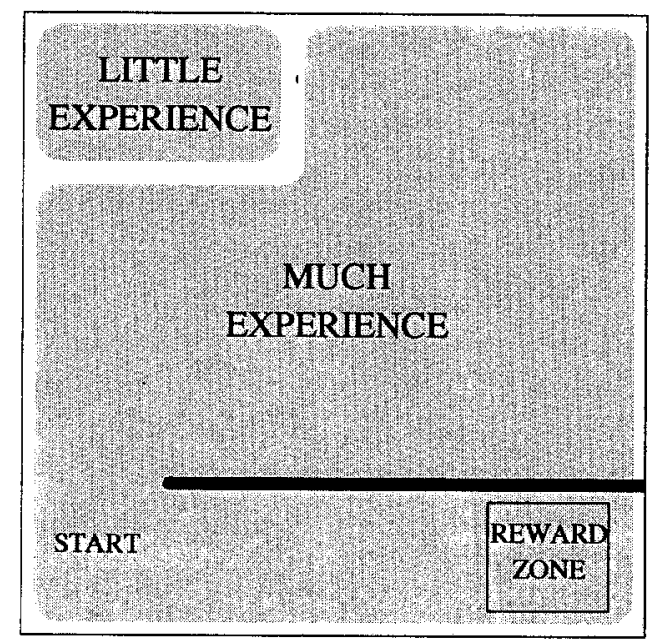

Figure 9. The state-space of a very simple path-planning problem. 
The selection of an appropriate $T_{\text {bored }}$ would be hard to formalize. It should take into account the following: the expected lifetime of the system, a measure of the importance of not becoming stuck during learning, and perhaps any available prior knowledge of the stochasticity of the system, or known constraints on the reward function. An automatic procedure for computing $T_{\text {bored }}$ would require a formal definition of the human programmer's requirements and a prior distribution of possible worlds.

\section{Experimental results}

This section begins with some comparative results in the familiar domain of stochastic two-dimensional maze worlds. It then examines the $\beta$ parameter, which specifies the amount of computation (number of Bellman equation backups) allowed per real-world observation, and also the $T_{\text {bored }}$ parameter, which defines how much exploration is performed. A number of larger examples are then used to investigate performance for a range of different discrete stochastic reinforcement tasks.

\section{Maze problems}

Each state has four actions: one for each direction. Blocked actions do not move. One goal state (the star in subsequent figures) gives 100 units of reward, all others give no reward, and there is a discount factor of 0.99 . Trials start in the bottom left corner. The system is reset to the start state whenever the goal state has been visited ten times since the last reset. The reset is outside the learning task: it is not observed as a state transition.

Dyna and prioritized sweeping were both allowed ten Bellman's equation backups per observation $(\beta=10)$. Two versions of Dyna were tested:

1. Dyna-PI+ is the original Dyna-P| of Sutton (1990), supplemented with the exploration bonus $(\epsilon=0.001)$ from the same paper.

2. Dyna-opt is the original Dyna-PI supplemented with the same $T_{\text {bored }}$ optimistic heuristic that is used to evaluate prioritized sweeping in this paper.

Table 2 shows the number of observations before convergence. A trial was defined to have converged by a given time if no subsequent sequence of 1000 decisions contained more than $2 \%$ suboptimal decisions. The test for optimality was performed by comparison with the control law obtained from full dynamic programming using the true simulation.

We begin with some results for deterministic problems, in the first three rows of table 2. The first row shows that Dyna-PI + converged for all problems except the 4528 state problem. A smaller exploration bonus than $e=0.001$ might have helped the latter problem converge, albeit slowly. The other two rows used the optimistic heuristic with $r^{\text {opt }}=$ 200 and $T_{\text {bored }}=1$. The $r^{\text {opt }}$ value thus overestimated the best possible reward by a factor of two-this was to see if we would converge without an accurate estimation of the true best possible reward. $T_{\text {bored }}=1$ meant that as soon as something was tried all optimism was lost. This is a safe strategy in a deterministic environment.

The learning controller was given no clues beyond those implicit in the two parameters $r^{\text {opt }}$ and $T_{\text {bored. }}$. Thus, to ensure convergence to the optimum, it had to sample each stateaction pair at least once. 
Table 2. Number of observations before $98 \%$ of decisions were subsequently optimal.

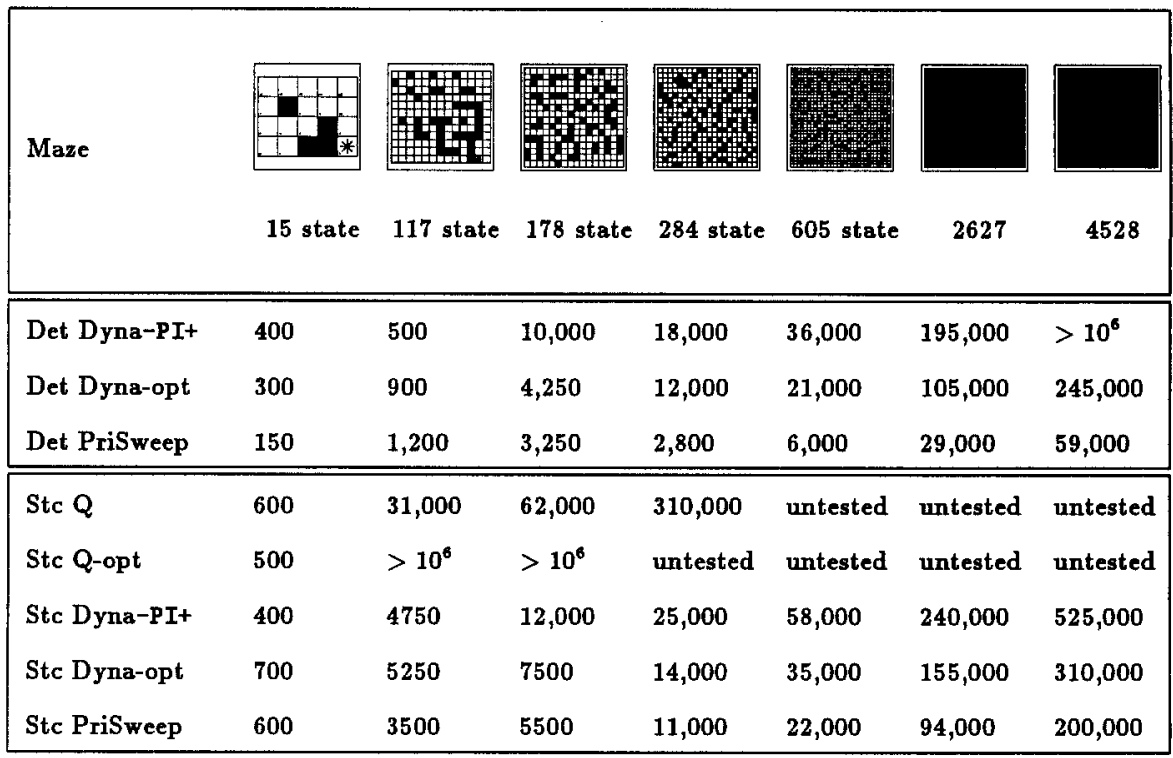

These values have been rounded. For prioritized sweeping (and Dyna, where applicable) $\beta=10, \epsilon=10^{-3}$, and $r^{\mathrm{opt}}=200$. The tabulated experiments were all only run once; however, further multiple runs of the optimistic Dyna and priortized sweeping have revealed little variance in convergence rate. See also figures 11 and 14 .

Prioritized sweeping required fewer steps than optimistic Dyna in all mazes but one small one. All learners and runs took between 10-30 seconds per thousand observations running on a Sun-4 workstation. Interestingly, prioritized sweeping usually took about half the real time of Dyna. This is because during much of the exploration there were so few surprises that it did not need to use its full allocation of Bellman's equation processing steps. This effect is even more pronounced if 300 processing steps per observation are allowed instead of ten. For example, in the 4528 state problem, optimistic Dyna then required 143,000 observations and took three hours. Prioritized sweeping required 21,000 observations and took 15 minutes.

The lower part of table 2 shows the results for stochastic problems using the same mazes. Each action had a $50 \%$ chance of being corrupted to a random value before it was applied. Thus if "North" was applied, the outcome was movement North $1 / 2+1 / 8=5 / 8$ of the time, and each other direction $1 / 8$ of the time. Prioritized sweeping and optimistic Dyna each used a $T_{\text {bored }}$ value of 5 . Thus, they sampled every state-action combination five times before losing their optimism. This value was chosen as a reasonable balance between exploration and exploitation, given the authors' knowledge of the stochasticity of the system, and happily it proved to be satisfactory. As we discussed in section 4.1 , the choice of $T_{\text {bored }}$ is not automated for any of these experiments.

These stochastic results also include a recent interesting incremental technique called Q-learning (Watkins, 1989), which manages to learn without constructing a state-transition model. Additionally, we tried Q-learning using the same $T_{\text {bored }}$ optimistic heuristic as prioritized sweeping. The initial $Q$ values were set high to encourage better initial exploration 
than a random walk. Much effort was put into tuning $Q$ for this application. Its performance was, however, worse. In particular, the optimistic heuristic is a disaster for Q-learning that easily gets trapped-this is because Q-learning only pays attention to the current state of the system, while the "planning to explore" behavior requires that attention is paid to areas of the state-space which the system is not currently in.

For the stochastic maze results, the difference between optmistic Dyna and prioritized sweeping is less pronounced. This is because the large number of predecessors quickly dilutes the wave of interesting changes that are propagated back on the priority queue, leading to a queue of many, very similar, priorities. However, prioritized sweeping still required less than half the total real time of either version of Dyna before convergence.

\section{A small, fully connected, example}

We also have results for a five-state benchmark problem described by Sato et al. (1988) and also used in Barto and Singh (1990). The transition matrix is in figure 10, and the results are shown in table 3 . A $T_{\text {bored }}$ parameter of 20 was used. In fact, $T_{\text {bored }}=5$ also converged 20 times out of 20, taking on average 120 steps and therefore $T_{\text {bored }}=20$ was considered a safe safety margin. The two Q-learners were extensively tweaked to find their best performance. The EQ-algorithm (Barto \& Singh, 1990) is designed to guarantee convergence at all costs-and so its poor comparative performance here is to be expected. Dyna-PI+ was given what was probably too small an exploration bonus for the problem. The reduced exploration meant faster convergence, but on one occasion some misleading early transitions caused it to get stuck with a suboptimal policy.

\begin{tabular}{|c|c|c|c|c|c|c|}
\hline & $\mathbf{R}$ & $\mathrm{n}=0$ & $\mathrm{n}=1$ & $\mathbf{n}=2$ & $n=3$ & $\mathrm{n}=4$ \\
\hline $\mathrm{sa}=0,0$ & -0.4 & 10 & 20 & 20 & 20 & 30 \\
\hline $\mathrm{sa}=0,1$ & 0 & 20 & 20 & 20 & 20 & 20 \\
\hline $\mathrm{sa}=0,2$ & 1.2 & 10 & 10 & 10 & 30 & 40 \\
\hline $\mathrm{sa}=1,0$ & -2.5 & 10 & 10 & 20 & 30 & 30 \\
\hline$s a=1,1$ & 0.2 & 10 & 10 & 60 & 10 & 10 \\
\hline $\mathrm{sa}=1,2$ & -1.3 & 10 & 50 & 20 & 10 & 10 \\
\hline $\mathrm{sa}=2,0$ & 1.5 & 10 & 40 & 20 & 20 & 10 \\
\hline $\mathrm{sa}=2,1$ & 0.8 & 30 & 20 & 20 & 20 & 10 \\
\hline $\mathrm{sa}=2,2$ & -1.4 & 30 & 20 & 10 & 10 & 30 \\
\hline $\mathrm{sa}=3,0$ & -1.2 & 20 & 50 & 10 & 10 & 10 \\
\hline $\mathbf{s a}=3,1$ & -0.7 & 30 & 10 & 10 & 40 & 10 \\
\hline $\mathrm{sa}=3,2$ & 0.1 & 20 & 30 & 30 & 10 & 10 \\
\hline $\mathrm{sa}=4,0$ & 2.4 & 20 & 20 & 20 & 20 & 20 \\
\hline $\mathrm{sa}=4,1$ & -1.7 & 20 & 30 & 20 & 10 & 20 \\
\hline $\mathrm{sa}=4,2$ & 1 & 10 & 40 & 20 & 10 & 20 \\
\hline
\end{tabular}

Figure 10. Transition probabilities $(\times 100)$ and expected rewards of a five-state, three-action, Markov control problem. $\mathrm{R}$ is the reward, $\mathrm{n}$ is the destination state, and sa indicates the current state and action. 
Table 3. The mean number of observations before $>98 \%$ of subsequent decisions were optimal.

\begin{tabular}{cccccc}
\hline$Q$ & Q-opt & Dyna-PI+ & Dyna-opt & PriSweep & EQ \\
\hline $2105 \pm 520$ & $2078 \pm 430$ & $\begin{array}{c}252 \pm 35 \\
\text { (one failure) }\end{array}$ & $470 \pm 21$ & $472 \pm 22$ & $7105 \pm 662$ \\
\hline
\end{tabular}

Note: Each learner was run 20 times and in all cases, bar one, there was eventual convergence to optimal performance. Also shown is the standard deviation of the 20 trials. The discount factor was $\gamma=0.8$. For the optimisitic methods, $r^{\mathrm{opt}}=10$ and $T_{\text {bored }}=20$. For priortized sweeping and Dyna $\beta=10$, and for priortized sweeping $\epsilon=10^{-3}$.

\section{The system parameters for prioritized sweeping}

We now look at two results to give insight into two important parameters of priortized sweeping. Firstly we consider its performance relative to the number of backups per observation. This experiment used the stochastic, 605 state example from table 2, and the results are graphed in figure 11. Using one operation is almost equivalent to optimistic Q-learning which does not converge. Even using only two backups gives reasonable performance, and performance improves as the number of backups increases. Beyond 50 backups, the priority queue usually gets exhausted on each time step, and there is little further improvement.

The other parameter is $T_{\text {bored }}$. We use a test case in which inadequate exploration is particularly dangerous. The maze in figure 12 has two reward states. The lesser reward of 50 comes from the state in the bottom right. The greater reward of 100 is from the more inaccessible state near the top right. Trials always begin from the bottom left, and the world is stochastic in the same manner as the earlier examples. Trials are reset when either goal state is encountered ten times. If $T_{\text {bored }}$ is set too low and if there is bad luck while attempting to explore near the large reward state, then the controller will lose interest, never return

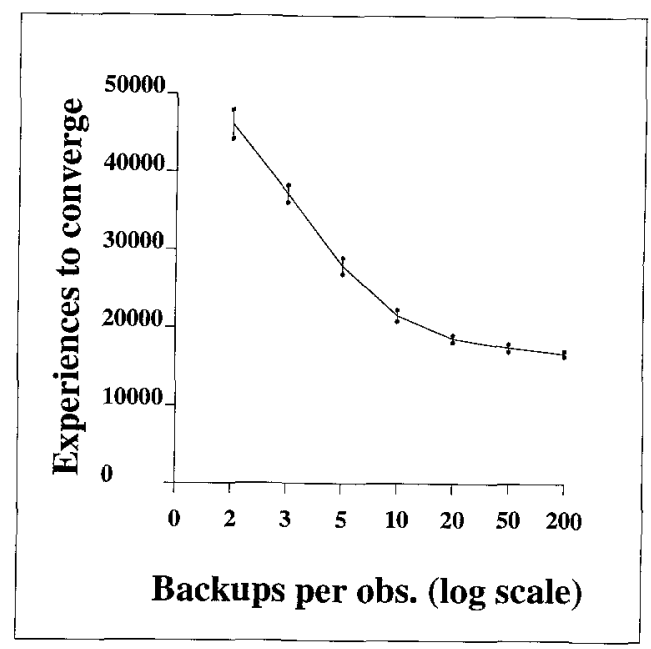

Fig. 11. Number of experiences needed for prioritized sweeping to converge, plotted against number of back-ups per observation $(\beta)$. This used the 605 state stochastic maze from table $2\left(\gamma=0.99, r^{\text {opt }}=200, T_{\text {bored }}=5\right.$, $\epsilon=10^{-3}$ ). The error bars show the standard deviations from ten runs with different random seeds. 


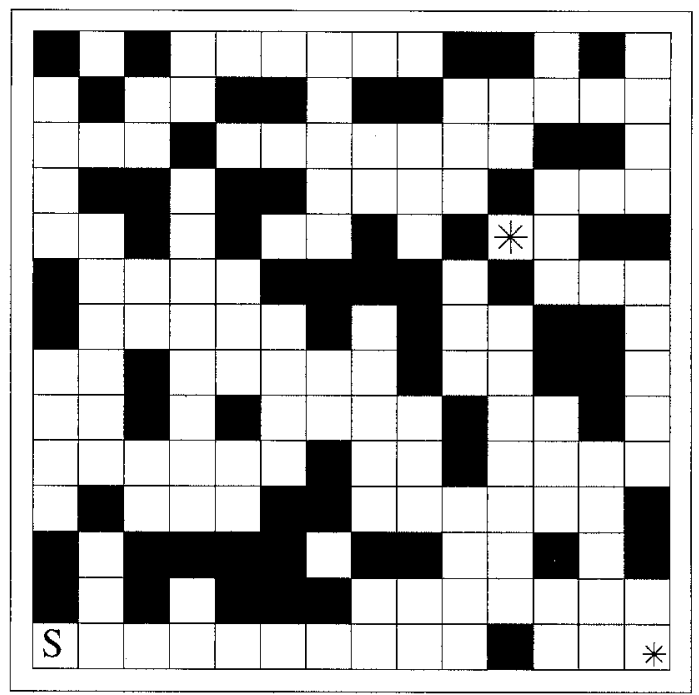

Figure 12. A misleading maze. A small reward in the bottom right tempts us away from a larger reward.

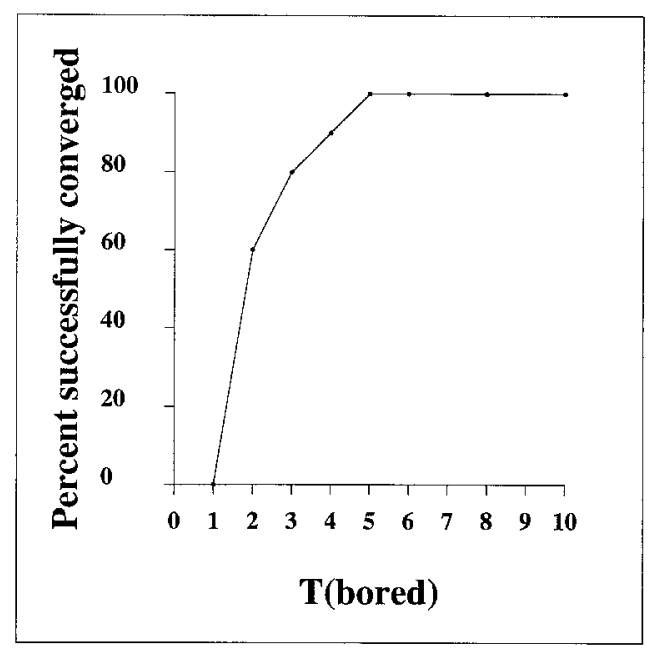

Figure 13. The frequency of correct convergence versus $T_{\text {bored }}$ for the misleading maze $\left(\gamma=0.99, r^{\mathrm{opt}}=\right.$ $200, \beta=10, \epsilon=10^{-3}$ ).

and very likely spend the rest of its days traveling to the inferior reward. Each value of $T_{\text {bored }}$ was run ten times, and we recorded the percentage of runs that had converged correctly by 50,000 observations. Figure 13 graphs the results. For this problem, $T_{\text {bored }}=5$ (which was checked a further 30 times) appears sufficient to ensure that we do not become stuck. 


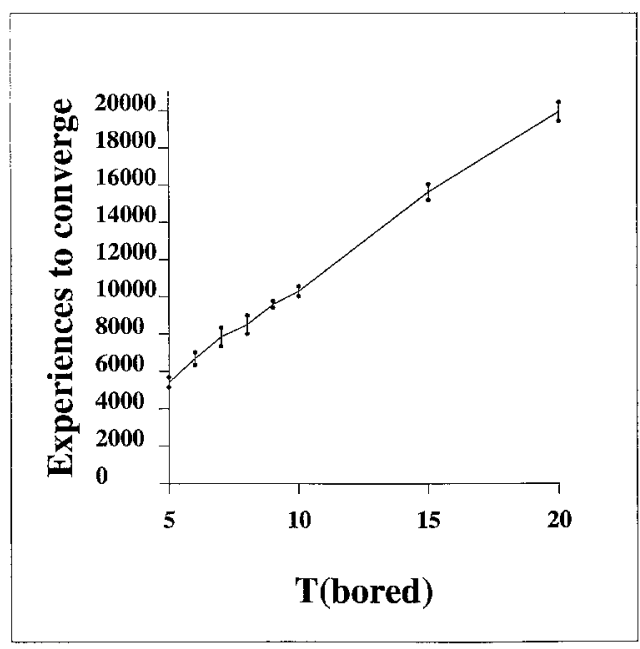

Figure 14. The mean and standard deviation of the number of experiences before convergence for ten independent experiments, as a function of $\mathbf{T}_{\text {bored }}$ for the misleading maze. Parameter values are as in figure 13.

Figure 14 shows the number of experiences needed for convergence as a function of $T_{\text {bored }}$ for the same set of experiments.

\section{Other tasks}

We begin with a task with a 3-D state-space quantized into 14,400 potential discrete states: guiding a rod through a planar maze by translation and rotation. There are four actions: move forward one unit along the rod's length, move backward one unit, rotate left one unit, and rotate right one unit. In fact, actions take us to the nearest quantized state. There are $20 \times 20$ position quantizations and 36 angle quantizations producing 14,400 states, though many are unreachable from the start configuration. The distance unit is $1 / 20$ th the width of the workspace, and the angular unit is $10^{\circ}$. The problem is deterministic but requires a long, very specific, sequence of moves to get to the goal. Figure 15 shows the problem, obstacles, and shortest solution for our experiments.

Q, Dyna-PI+, Optimistic Dyna, and prioritized sweeping were all tested. The results are in table 4.

$\mathrm{Q}$ and Dyna-PI+ did not even travel a quarter of the way to the goal, let alone discover an optimal path, within 200,000 experiences. It is possible that a very well-chosen exploration bonus would have helped Dyna-PI+, but in the four different experiments we tried, no value produced stable exploration.

Optimistic Dyna and prioritized sweeping both eventually converged, with the latter requiring a third the experiences and a fifth the real time.

When 2000 backups per experience were permitted, instead of 100, then both optimistic Dyna and prioritized sweeping required fewer experiences to converge. Optimistic Dyna took 21,000 experiences instead of 55,000 but took 2900 seconds-almost twice the real 


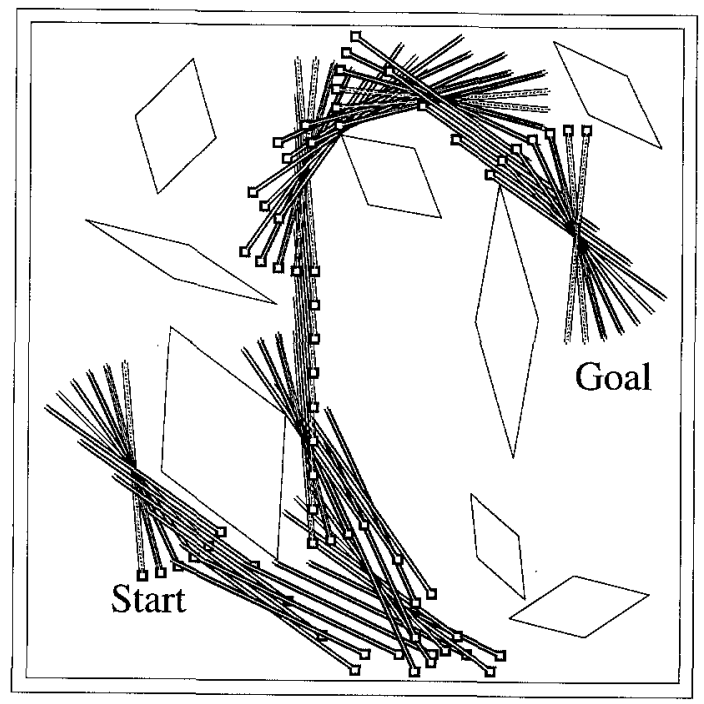

Figure 15. A three-DOF problem and the shortest solution path.

Table 4. Performance on the deterministic rod-in-maze task.

\begin{tabular}{lcc}
\hline & Experiences to converge & Real time to converge \\
\hline $\mathrm{Q}$ & Never \\
Dyna-PI+ & Never & \\
Optimistic Dyna & 55,000 & 1500 secs \\
Prioritized Sweeping & 14,000 & 330 secs \\
\hline Both Dynas and priortized sweeping were allowed 100 backups per experience $(\gamma$ \\
$=0.99, \quad r^{\text {opt }}=200, \beta=100, T_{\text {bored }}=1, \epsilon=10^{-3)}$.
\end{tabular}

time. Prioritized sweeping took 13,500 instead of 14,000 experiences-very little improvement, but it used no extra time. This indicates that for prioritized sweeping, 100 backups per observation is sufficient to make almost complete use of its observations, so that all the long-term reward $\left(\hat{J}_{i}\right)$ estimates are very close to the estimates that would be globally consistent with the transition probability estimates $\left(\hat{q}_{i j}^{a}\right)$. Thus, we conjecture that even full dynamic programming after each experience (which would take days of real time) would do little better.

We also consider a more complex extension of the maze world, invented by Singh (1991), which consists of a maze and extra state information dependent on where you have visited so far in the maze. We use the example in figure 16. There are 263 cells, but there are also four binary flags appended to the state, producing a total of $263 \times 16=4208$ states. The flags, named A, B, C, and X, are set whenever the cell containing the corresponding letter is passed through. All flags are cleared when the start state (in the bottom left-hand corner) is entered. A reward is given when the goal state (top right) is entered, only if flags $\mathrm{A}, \mathrm{B}$, and $\mathrm{C}$ are set. Flag $\mathrm{X}$ provides further interest. If $\mathrm{X}$ is clear, the reward is 


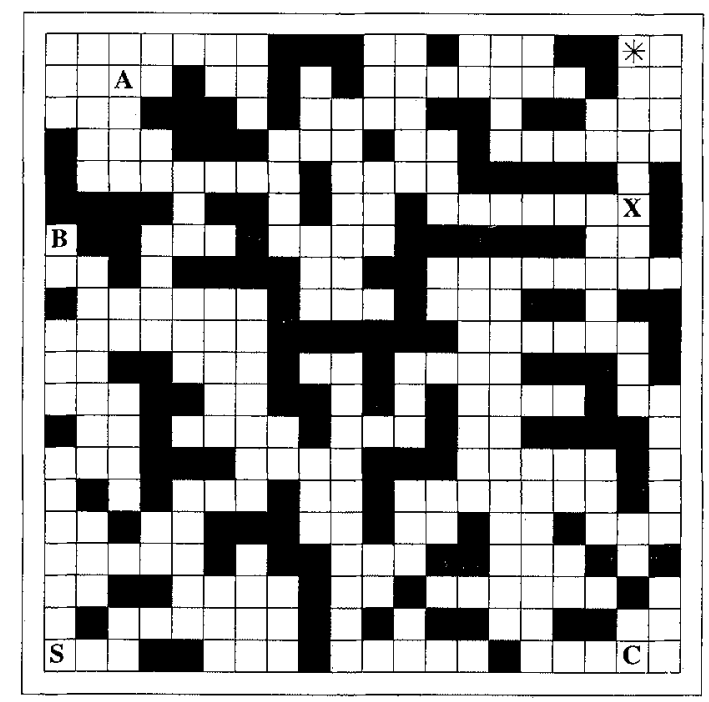

Figure 16. A maze with extra state in the form of four binary flags.

100 units. If $\mathrm{X}$ is set, the reward is only 50 units. This task does not specify in which order A, B, and C are to be visited. The controller must find the optimal path.

Prioritized sweeping was tried with both the deterministic and stochastic maze dynamics $\left(\gamma=0.99, r^{\mathrm{opt}}=200, \beta=10, \epsilon=10^{-3}\right)$. In the deterministic case, $T_{\text {bored }}=1$. In the stochastic case, $T_{\text {bored }}=5$. In both cases it found the globally optimal path through the three good flags to the goal, avoiding flag $X$. The deterministic case took 19,000 observations and 20 minutes of real time. The stochastic case required 120,000 observations and two hours of real time.

In these experiments, no information regarding the special structure of the problem was available to the learner. For example, knowledge of the cell at coordinates $(7,1)$ with flag A set had no bearing on knowledge of the cell at coordinates $(7,1)$ with A clear. If we told the learner that cell transitions were independent of flag settings, then the convergence rate would be increased considerably. A far more interesting possibility is the automatic discovery of such structure by inductive inference on the structure of the learned state transition matrix. See Singh (1991) for current interesting work in that direction.

The third experiment is the familiar pole-balancing problem of Michie and Chambers (1968). There is no place here to discuss the enormous number of techniques that have been applied to this problem along with an equally enormous variation in details of the task formulation. The state-space of the cart is quantized at three equal levels for cart position, cart velocity, and pole angular speed. It is quantized at six equal levels for pole angle. The simulation used four real-valued state variables, yet the learner was only allowed to base its control decisions on the current quantized state. There are two actions: thrust left $10 \mathrm{~N}$ and thrust right $10 \mathrm{~N}$. The problem is interesting because it involves hidden state-the controller believes the system is Markov when in fact it is not. This is because there are many possible values for the real-valued state variables in each discretized box, and 
successor boxes are partially determined by these real values, which are not given to the controller. The task is defined by a reward of 100 units for every state except one absorbing state corresponding to a crash, which receives zero reward. Crashes occur if the pole angle or cart position exceed their limits. A discount factor of $\gamma=0.999$ is used, and trials start in random survivable configurations. Other parameters are $\left(r^{\mathrm{opt}}=200, \beta=100\right.$, $T_{\text {bored }}=1, \epsilon=10^{-3}$ ).

If the simulation contains no noise, or a very small amount $(0.1 \%$ added to the simulated thrust), prioritized sweeping very quickly (usually in under 1000 observations and 15 crashes) develops a policy that provides stability for approximately 100,000 cycles. With a small amount of noise (1\%), stable runs of approximately 20,000 time steps are discovered after, on average, 30 crashes.

\section{Heuristics to guide search}

In all experiments to date, the optimistic estimate of the best available one-step reward, $r^{\text {opt }}$, has been set to an overestimate of the best reward that is actually available. However, if the human programmer knows in advance what is the best possible reward-to-go from any given state, then the resultant, more realistic, optimism does not need to experience all state-action pairs.

For example, consider the maze world. If the robot is told the location of the goal state (in all previous experiments it was not given this information), but is not told which states are blocked, then it can nevertheless compute what would be the best possible reward-togo from a state. It could not be greater than the reward obtained from the shortest possible path to the goal. The length of the path, $l$, can be computed easily with the Manhattan distance metric, and then the best possible reward-to-go is

$$
0 \gamma^{1}+0 \gamma^{2}+\ldots+0 \gamma^{l-1}+r^{\mathrm{opt}} \gamma^{1}+r^{\mathrm{opt}} \gamma^{l+1}+\ldots=\frac{r^{\mathrm{opt}} \gamma^{l}}{1-\gamma}
$$

When this optimistic heuristic is used, initial exploration is biased towards the goal, and once a path is discovered then many of the unexplored areas may be ignored. Ignoring occurs when even the most optimistic reward-to-go of a state is no greater than that of the already obtained path.

For example, figure 17 shows the areas explored using a Manhattan heuristic when finding the optimal path from the start state at the bottom leftmost cell to the goal state at the center of the maze. The maze has 8525 states of which only 1722 needed to be explored.

For some tasks we may be satisfied to cease exploration when we have obtained a solution known to be, say, within $50 \%$ of the optimal solution. This can be achieved by using a heuristic which lies: it tells us that the best possible reward-to-go is that of a path that is twice the length of the true shortest possible path. 


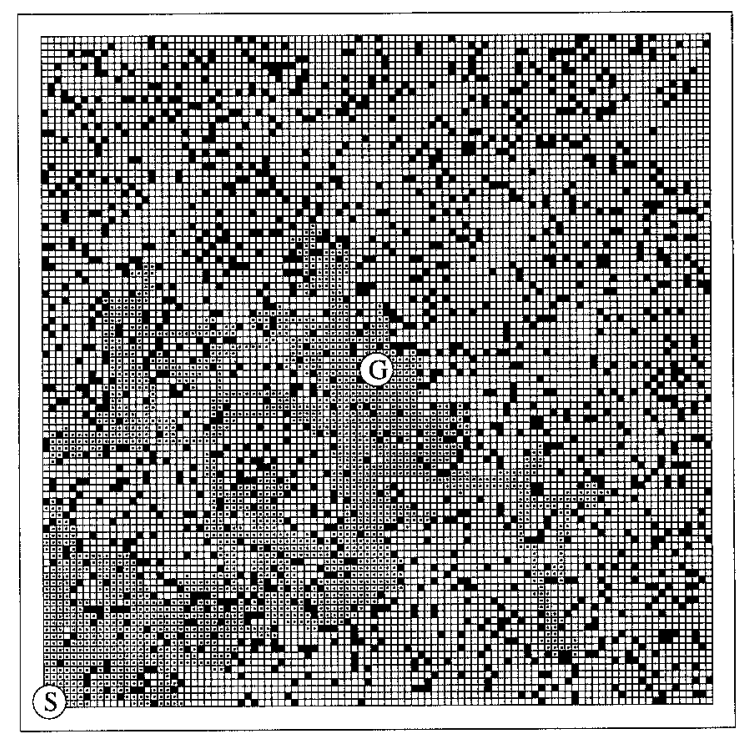

Figure 17. Dotted states are all those visited when the Manhattan heuristic was used to derive $r^{\text {opt }}(\gamma=0.99$, $\beta=10, T_{\text {bored }}=1, \epsilon=10^{-3}$ ).

\section{Discussion}

\section{Generalization of the state transition model}

This article has been concerned with discrete state systems in which no prior assumptions are made about the structure of the state-space. Despite the weakness of the assumptions, we can successfully learn large stochastic tasks. However, very many problems do have extra known structure, and it is important to consider how this knowledge can be used. By far the most common knowledge is smoothness-given two states that are in some way similar, in general their transition probabilities will be similar.

TD can also be applied to highly smooth problems using a parametric function approximator such as a neural network. This technique has recently been used successfully on a large complex problem, backgammon, by Tesauro (1991). The discrete version of prioritized sweeping given in this article could not be applied directly to backgammon because the game has $10^{23}$ states, which is unmanageably large by a factor of at least $10^{10}$. However, a method that quantized the space of board positions, or used a more sophisticated smoothing mechanism, might conceivably be able to compute a near-optimal strategy.

We are currently developing memory-based algorithms that take advantage of local smoothness assumptions. In these investigations, state transition models are learned by memory-based function approximators (Moore \& Atkeson, 1992). Prioritized sweeping takes place over non-uniform tessellations of state-space, partitioned by variable resolution $k$ d-trees (Moore, 1991). We are also investigating the role of locally linear control rules and reward functions in such partitionings, in which instead of using Bellman's equation 
(16) directly, we use local linear quadratic regulators (LQR) (see, for example, Sage \& White, 1977). It is worth remembering that, if the system is sufficiently linear, $L Q R$ is an extremely powerful technique. In a pole-balancer experiment in which we used local weighted regression to identify a local linear model, LQR was able to create a stable controller based on only 31 state transitions!

Other current investigations that attempt to perform generalization in conjunction with reinforcement learning are Mahadevan and Connell (1990), which investigates clustering parts of the policy; Chapman and Kaelbling (1990), which investigates automatic detection of locally relevant state variables; and Singh (1991), which considers how to automatically discover the structure in tasks such as the multiple-flags example of figure 16.

\subsection{Related work}

\section{The Queue-Dyna algorithm of Peng and Williams}

Peng and Williams (1992) have concurrently been developing a closely related algorithm that they call Queue-Dyna. This conceptually similar idea was discovered independently. Where prioritized sweeping provides efficient data processing for methods that learn the state-transition model, Queue-Dyna performs the same role for Q-learning (Watkins, 1989), an algorithm that avoids building an explicit state-transition model. Queue-Dyna is also more careful about what it allows onto the priority queue: it only allows predecessors that have a predicted change ("interestingness" value) greater than a significant threshold $\delta$, whereas prioritized sweeping allows everything above a minuscule change $\left(\epsilon=10^{-5}\right.$ times the maximum reward) onto the queue. The initial experiments in Peng and Williams (1992) consist of sparse, deterministic maze worlds of several hundred cells. Performance, measured by the total number of Bellman's equation processing steps before convergence, is greatly improved over conventional Dyna-Q (Sutton, 1990).

\section{Other related work}

Sutton (1990) identifies reinforcement learning with asynchronous dynamic programming and introduces the same computational regime as that used for prioritized sweeping. The notion of using an optimistic heuristic to guide search goes back to the $A^{*}$ tree search algorithm of Nilsson (1971), which also motivated another aspect of prioritized sweeping: it too schedules nodes to be expanded according to an (albeit different) priority measure. More recently, Korf (1990) gives a combination of $A^{*}$ and Dynamic Programming in the LRTA* algorithm. LRTA* is, however, very different from prioritized sweeping: it concentrates all search effort on a finite-horizon set of states beyond the current actual system state. Finally, Lin (1991) has investigated a simple technique that replays, backwards, the memorized sequence of experiences that the controller has recently had. Under some circumstances, this may produce some of the beneficial effects of prioritized sweeping.

\section{Conclusion}

Our investigation shows that prioritized sweeping can solve large state-space real-time problems with which other methods have difficulty. Other benefits of the memory-based 
approach, described in Moore and Atkeson (1992), allow us to control forgetting in potentially changeable environments and to automatically scale state variables. Prioritized sweeping is heavily based on learning a world model, and we conclude with a few words on this topic.

If a model of the world is not known to the human programmer in advance, then an adaptive system is required, and there are two alternatives:

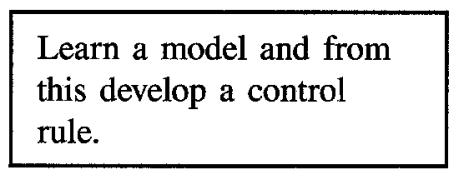

\begin{tabular}{|l|}
\hline $\begin{array}{l}\text { Learn a control rule } \\
\text { without building a } \\
\text { model. }\end{array}$ \\
\hline
\end{tabular}

Dyna and prioritized sweeping fall into the first category. Temporal differences and Qlearning fall into the second. Two motivations for not learning a model are 1) the interesting fact that the methods do, nevertheless, learn, and 2) the possibility that this more accurately simulates some kinds of biological learning (Sutton and Barto, 1990). However, a third advantage that is sometimes touted-that there are computational benefits in not learning a model-is, in our view, dubious. A common argument is that with the real world available to be sensed directly, why should we bother with less reliable, learned internal representations? The counterargument is that even systems acting in real time can, for every one real experience, sample millions of mental experiences from which to make decisions and improve control rules.

Consider a more colorful example. Suppose the anti-model argument was applied by a new arrival at a university campus: "I don't need a map of the university-the university is its own map." If the new arrival truly mistrusts the university cartographers, then there might be an argument for one full exploration of the campus in order to create his or her own map. However, once this map has been produced, the amount of time saved overall by pausing to consult the map before traveling to each new location-rather than exhaustive or random search in the real world-is undeniably enormous.

It is justified to complain about the indiscriminate use of combinatorial search or matrix inversion prior to each supposedly real-time decision. However, models need not be used in such an extravagant fashion. The prioritized sweeping algorithm is just one example of a class of algorithms that can easily operate in real time and also derive great power from a model.

\section{Acknowledgments}

Many thanks to Mary Lee, Rich Sutton and the reviewers of this article for some very valuable comments and suggestions. Thanks also to Stefan Schaal and Satinder Singh for useful comments on an early draft. Andrew W. Moore is supported by a Postdoctoral Fellowship from SERC/NATO. Support was also provided under Air Force Office of Scientific Research grant AFOSR-89-0500, an Alfred P. Sloan Fellowship, the W.M. Keck Foundation Associate Professorship in Biomedical Engineering, Siemens Corporation, and a National Science Foundation Presidential Young Investigator Award to Christopher G. Atkeson. 


\section{Appendix: The random generation of a stochastic problem}

Here is an algorithm to generate stochastic systems such as figure 4 in section 3 . The parameters are $S_{n t}$, the number of non-terminal states; $S_{t}$, the number of terminal states; and $\mu_{\text {succs }}$, the mean number of successors.

All states have a position within the unit square. The terminal states are generated on an equispaced circle, diameter 0.9 , alternating between black and white. Non-terminal states are each positioned in a uniformly random location within the square. Then the successors of each non-terminal are selected. The number of successors is chosen randomly as $1+$ $X$ where $X$ is a random variable drawn from the exponential distribution with mean $\mu_{\text {succs }}$ -1 .

The choice of successors is affected by locality within the unit square. This provides a more interesting system than allowing successors to be entirely random. It was empirically noted that entirely random successors cause the long-term absorption probabilities to be very similar across most of the set of states. Locality leads to a more varied distribution.

The successors are chosen according to a simple algorithm in which they are drawn from within a slowly growing circle centered on the parent state.

If the parent state is $i$, and there are $N_{i}$ successors, then the $j$ th transition probability is computed by $X_{j} /\left(\Sigma_{k=1}^{N i} X_{k}\right)$, where $\left\{X_{1}, \ldots, X_{N i}\right\}$ are independent random variables, uniformly distributed in the unit interval.

Once the system has been generated, a check is performed that the system is absorbingall non-terminals can eventually reach at least one terminal state. If not, entirely new systems are randomly generated until an absorbing Markov system is obtained.

\section{References}

Barto, A.G., \& Singh, S.P. (1990). On the computational economics of reinforcement learning. In D.S. Touretzky, J.L. Elman, T.J. Sejnowski, and G.E. Huiton (Eds.), Connectionist Models: Proceedings of the 1990 Summer School. San Mateo, CA: Morgan Kaufmann (pp. 35-44).

Barto, A.G., Sutton, R.S., \& Watkins, C.J.C.H. (1989). Learning and sequential decision making (COINS Technical Report 89-95). Amherst, MA: University of Massachusetts.

Barto, A.G., Bradtke, S.J., \& Singh, S.P. (1991). Real-time learning and control using asynchronous dynamic programming (COINS Technical Report 91-57). Amherst, MA: University of Massachusetts.

Bellman, R.E. (1957). Dynamic programming. Princeton, NJ: Princeton University Press.

Berry, D.A., \& Fristedt, B. (1985). Bandit problems: Sequential allocation of experiments. New York, NY: Chapman and Hall.

Bertsekas, D.P., \& Tsitsiklis, J.N. (1989). Parallel and distributed computation. Englewood Cliffs, NJ: Prentice Hall.

Chapman, D., \& Kaelbling, L.P. (1990). Learning from delayed reinforcement in a complex domain (Technical Report No. TR-90-11). Teleos Research, Palo Alto, CA.

Christiansen, A.D., Mason, M.T., \& Mitchell, T.M. (1990). Learning reliable manipulation strategies without initial physical models. In IEEE Conference on Robotics and Automation (pp. 1224-1230). IEEE Computer Society Press, Washington, DC.

Dayan, P. (1992). The convergence of $\operatorname{TD}(\lambda)$ for general $\lambda$. Machine Learning, 8(3), 341-362.

Kaelbling, L.P. (1990). Learning in embedded systems. PhD. thesis, Department of Computer Science, Stanford University, Stanford CA. (Technical Report No. TR-90-04.)

Knuth, D.E. (1973). Sorting and searching. Reading, MA: Addison Wesley.

Korf, R.E. (1990). Real-time heuristic search. Artificial Intelligence, 42, 189-211. 
Lin, L.J. (1991). Programming robots using reinforcement learning and teaching. In Proceedings of the Ninth International Conference on Artificial Intelligence (AAAI-91). Cambridge, MA: MIT Press.

Mahadevan, S., \& Connell, J. (1990). Automatic programming of behavior-based robots using reinforcement learning (Technical Report). IBM T.J. Watson Research Center. Yorktown Heights, NY.

Michie, D., \& Chambers, R.A. (1968). BOXES: An experiment in adaptive control. In E. Dale and D. Michie (Eds.), Machine intelligence 2. London: Oliver and Boyd, pp. 137-152.

Moore, A.W. \& Atkeson, C.G. (1992). Memory-based function approximators for learning control. In preparation.

Moore, A.W. (1991). Variable resolution dynamic programming: efficiently learning action maps in multivariate real-valued state-spaces. In L. Birnbaum \& G. Collins (Eds.), Machine learning: Proceedings of the eighth intemational workshop. San Mateo, CA: Morgan Kaufman, pp. 333-337.

Nilsson, N.J. (1971). Problem solving methods in artificial intelligence. New York: McGraw Hill.

Peng, J. \& Williams, R.J. (1992). Efficient search control in Dyna. College of Computer Science, Northeastern University, Boston, MA. (A revised version will appear as "Efficient learning and planning within the dyna framework." Proceedings of the Second International Conference on Simulation of Adaptive Behavior. Cambridge, MA: MIT Press, 1993.)

Sage, A.P., \& White, C.C. (1977). Optimum systems control. Englewood Cliffs, NJ: Prentice Hall.

Samuel, A.L. (1959). Some studies in machine learning using the game of checkers. IBM Journal on Research and Development, 3, (3)210-229. Reprinted in E.A. Feigenbaum \& J. Feldman (Eds.). (1963). Computers and thought. New York: McGraw-Hill, pp. 71-105.

Sato, M., Abe, K., \& Takeda, H. (1988). Learning control of finite Markov chains with an explicit trade-off between estimation and control. IEEE Transactions on Systems, Man, and Cybernetics, 18(5), 667-684.

Singh, S.P. (1991). Transfer of learning across compositions of sequential tasks. In L. Birnbaum \& G. Collins (EDs.). Machine learning: Proceedings of the eighth international workshop. Morgan Kaufman, pp. 348-352.

Stanfill, C. \& Waltz, D. (1986). Towards memory-based reasoning. Communications of the ACM, 29(12), 1213-1228.

Sutton, R.S., \& Barto, A.G. (1990). Time-derivative models of Pavlovian reinforcement. In M. Gabriel \& J. Moore (Eds.), Learning and computational neuroscience: Foundations of adaptive networks (pp. 497-537). Cambridge, MA: MIT Press.

Sutton, R.S. (1984). Temporal credit assignment in reinforcement learning. Ph.D. thesis, Department of Computer and Information Sciences, University of Massachusetts, Amherst.

Sutton, R.S. (1988). Learning to predict by the methods of temporal differences. Machine Learning, 3, 9-44.

Sutton, R.S. (1990). Integrated architecture for learning, planning, and reacting based on approximating dynamic programming. In Proceedings of the 7th International Conference on Machine Learning. San Mateo, CA: Morgan Kaufman.

Tesauro, G.J. (1991). Practical issues in temporal difference learning. Report RC 17223 (76307). IBM T.J. Watson Research Center. Yorktown Heights, NY.

Thrun, S.B., \& Möller, K. (1992). Active exploration in dynamic environments. In J.E. Moody, S.J. Hanson, \& R.P. Lippman (Eds.), Advances in neural information processing systems 4. San Mateo, CA: Morgan Kaufmann, pp. 531-538.

Watkins, C.J.C.H. (1989). Leaming from delayed rewards. Ph.D. thesis, King's College, University of Cambridge, United Kingdom.

Received July 23, 1992

Accepted September 22, 1992

Final Manuscript December 18, 1992 